\title{
The Next Generation Advanced Video Guidance Sensor: Flight Heritage and Current Development
}

\author{
Richard T. Howard and Thomas C. Bryan \\ Automated Rendezvous and Docking Development and Test Branch (ES62) \\ NASA - Marshall Space Flight Center, Huntsville, AL 35812 \\ 256-544-3536; ricky.howard@nasa.gov
}

\begin{abstract}
The Next Generation Advanced Video Guidance Sensor (NGAVGS) is the latest in a line of sensors that have flown four times in the last 10 years. The NGAVGS has been under development for the last two years as a long-range proximity operations and docking sensor for use in an Automated Rendezvous and Docking (AR\&D) system. The first autonomous rendezvous and docking in the history of the U.S. Space Program was successfully accomplished by Orbital Express, using the Advanced Video Guidance Sensor (AVGS) as the primary docking sensor. That flight proved that the United States now has a mature and flight proven sensor technology for supporting Crew Exploration Vehicles (CEV) and Commercial Orbital Transport Systems (COTS) Automated Rendezvous and Docking (AR\&D). NASA video sensors have worked well in the past: the AVGS used on the Demonstration of Autonomous Rendezvous Technology (DART) mission operated successfully in "spot mode" out to $2 \mathrm{~km}$, and the first generation rendezvous and docking sensor, the Video Guidance Sensor (VGS), was developed and successfully flown on Space Shuttle flights in 1997 and 1998.

This paper presents the flight heritage and results of the sensor technology, some hardware trades for the current sensor, and discusses the needs of future vehicles that may rendezvous and dock with the International Space Station (ISS) and other Constellation vehicles. It also discusses approaches for upgrading AVGS to address parts obsolescence, and concepts for minimizing the sensor footprint, weight, and power requirements. In addition, the testing of the various NGAVGS development units will be discussed along with the use of the NGAVGS as a proximity operations and docking sensor.
\end{abstract}

Keywords: Sensors, Docking, Automated, Proximity Operations, Relative Navigation, Rendezvous, Video PACS: 07.07.Df

\section{INTRODUCTION}

The Next Generation Advanced Video Guidance Sensor (NGAVGS) has been under development at NASA's Marshall Space Flight Center (MSFC) for the last three years, but the basic sensor technology has flown on three different spacecraft over the last 10 years and has been developed over the last 20 years. That latest mission was the 2007 Orbital Express (OE) mission that consisted of a pair of spacecraft outfitted with all of the hardware and software necessary to demonstrate the technical feasibility of different operations necessary for performing satellite servicing. The different operations performed during the $\mathrm{OE}$ mission were all completely automated and consisted of spacecraft rendezvous, spacecraft proximity operations, spacecraft docking, spacecraft free-flyer capture, fluid transfers, and Orbital Replacement Unit (ORU) transfers. Prior to OE, the sensor flew on the Demonstration of Autonomous Rendezvous Technologies (DART) mission in 2005. And in 1997 and 1998, the sensor technology was flown on the Space Shuttle and used to track the Spartan 301. In each of these missions, the sensor performed extremely well, despite the challenges of the environment and the varied orbital lighting conditions.

The sensor development has proceeded to both reproduce the functionality of the previous sensors (acquiring and tracking targets at ranges of up to 300 meters), but the sensor development has also gone beyond that in order to be able to detect retro-reflectors at ranges of up to 5000 meters. In addition, at very long ranges, the sensor software 
has been modified to allow limited three-degree-of-freedom tracking, providing bearing and range data. In addition, radiation was a concern for the new sensor, so parts were chosen to minimize the risk of radiation damage or upsets. Shown below in Figure 1 is the culmination of this work, completed in late October, 2008.

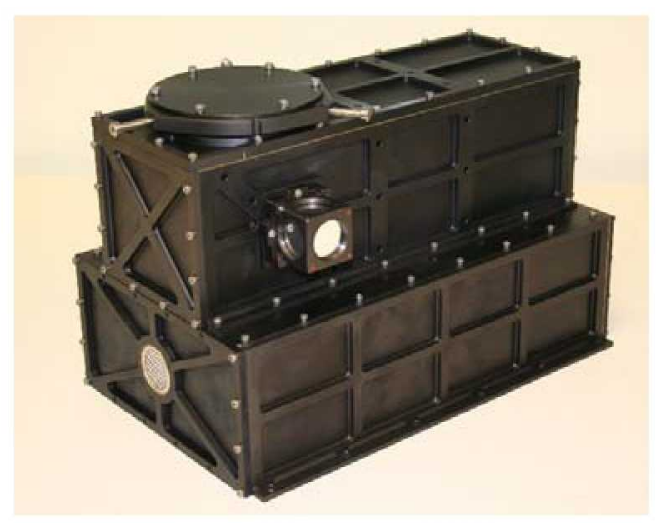

FIGURE 1. Next Generation Advanced Video Guidance Sensor (NGAVGS) Block II Prototype

\section{NGAVGS DEVELOPMENT}

Building on the AVGS laser illumination and imaging technology, the NGAVGS consists of two sets of laser diodes which operate at nominal wavelengths of 806 and $845 \mathrm{~nm}$, a mirror through which the lasers fire, a camera that images the return from the lasers, and hardware, software, and firmware that process the returned images into relative position and attitude data. The sensor is designed to interact with a retro-reflective target. The target has filters that allow one wavelength of AVGS laser to pass through, while blocking the other wavelength. That arrangement causes reflections from the target to occur when one set of lasers is illuminating it, but not when the other set is. The target retro-reflectors are arranged in a pattern known to the AVGS software. The sensor fires the lasers that are passed by the filters and captures an image, and then it fires the second set of lasers and captures a second image. When this second image is subtracted from the first image and an intensity threshold is used, virtually all of the background clutter is eliminated. The remaining lit pixel data is converted into a set of spots, and the spots are compared to the target pattern. Once a set of spots matching the target is found, the software computes the relative position and attitude between the target and the sensor. This data is output from the sensor and fed to the spacecraft Guidance and Navigation System.

As an in-house project, the NGAVGS hardware and software design is being accomplished by the MSFC Engineering Directorate personnel. An initial study was conducted in late 2006 to assess 13 different configuration options for the NGAVGS. The recommendation resulting from this trade was a two box configuration with a remote camera head that would be relatively inexpensive and would be mounted outside the spacecraft and a laser/electronics box that could be internally mounted in the spacecraft to provide radiation protection and thermal dissipation. An NGAVGS brassboard was built based on this configuration, with two different imaging heads to allow for side-by-side performance comparison, and it is depicted below in Figure 2.

There are several modes of operation for the NGAVGS. The primary NGAVGS modes of operation, based on the OE AVGS modes are as follows: 1) Standby (in which the sensor sends out status messages while awaiting further commands), 2) Acquisition (in which the sensor is commanded to actively seek a target and go into Tracking once a valid target is found), and 3) Tracking (the sensor is actively tracking a target that was found during Acquisition). More information about AVGS can be found in other publications (Howard et al., 2004).

An initial application for the AR\&D sensor technology for station will be to support the COTS for re-supply to ISS utilizing ISS/JEM and Node 2 hemispherical targets. The NGAVGS will be able to guide a spacecraft into range to support berthing applications with ISS using the existing ISS Long Range Targets (LRT). As the ISS does not currently have Short Range Targets (SRTs), SRTs would need to be located on ISS prior to docking applications. MSFC has done numerous target developments and in this application, a target layout would need to be configured to coordinate with the target geometry of the NGAVGS software/firmware. Testing would need to be completed 
prior to integration with ISS. ISS hemispherical retro-reflector and an SRT are both shown below in Figure 3 and are utilized in the NGAVGS Optical Characterization Tests (OCTs). Target development is key to the successful use of a sensor for AR\&D. For each Constellation Design Reference Mission (DRM) target layout needs to be assessed and tested because it is integral to the sensor performance.

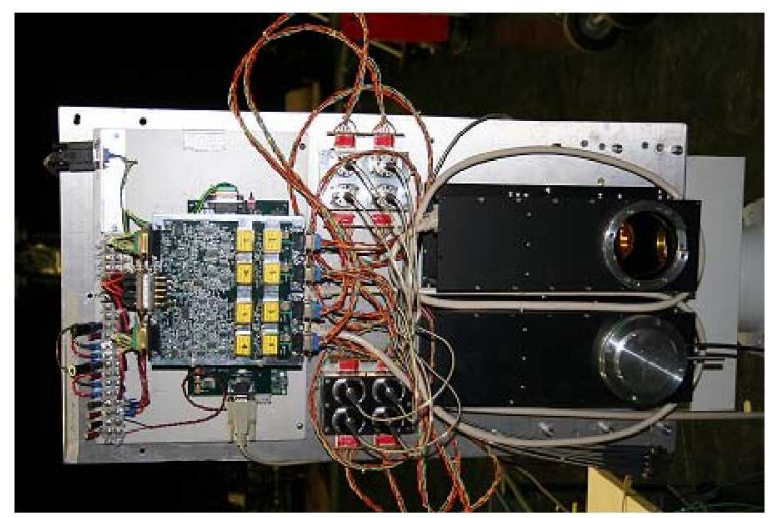

FIGURE 2. Brassboard configuration of NGAVGS

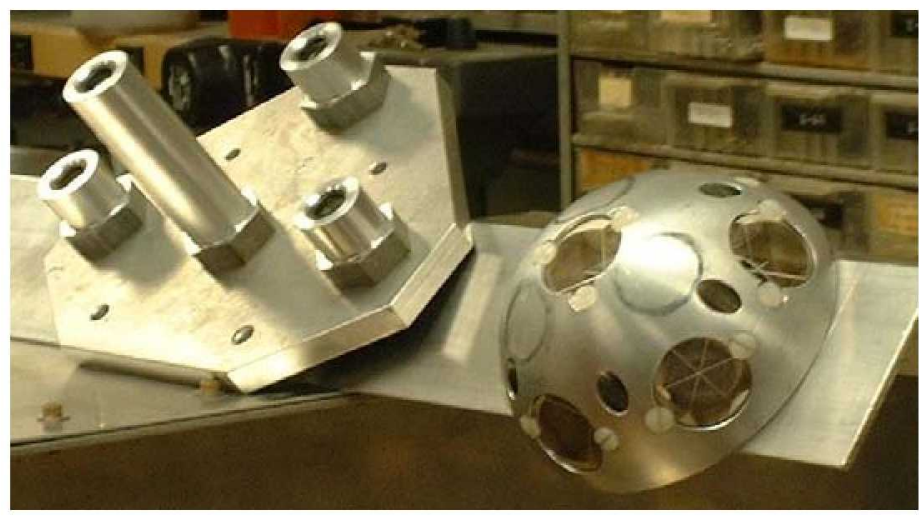

FIGURE 3. ISS/JEM Hemispherical Target and Short Range Target

\section{NGAVGS SENSOR SPECIFICATIONS}

The weight, volume, and power consumption estimates for the NGAVGS are on the order of the AVGS values. The AVGS weighed $20 \mathrm{lbs}$, was approximately $7 \times 10 \times 12$ inches, and consumed approximately 14 Watts in Standby mode and 35 Watts in Tracking Mode (the most power intensive mode of operation). It became clear that to meet the needs of the various mission applications, the MSFC team needed to develop more flexibility into the NGAVGS sensor design packaging. As a result, there is a one box configuration which minimizes the weight and physical integration requirements and a two box design that allows the sensor head to be mounted externally and the laser and electronics box to be mounted internally to reduce environmental effects. The two box concept is shown in Figure 4 below. The NGAVGS one box design is approximately $7 \times 7.5 \times 12$ inches and weighs approximately $8 \mathrm{~kg}(17.5$ pounds). It consumes an average of less than $30 \mathrm{~W}$ during tracking operations (the most power intense mode of operation). The NGAVGS one box design is shown in Figure 1, and that unit is the second prototype unit built, and the NGAVGS two-box concept is shown in Figure 4 below.

The NGAVGS functions in a fashion similar to the AVGS - it takes two sequential pictures illuminated by different wavelengths of laser light, subtracts one picture from the other, creates spots from the lit pixel image data, matches spots to the known target geometry, and computes the relative position vector and relative attitude information. 


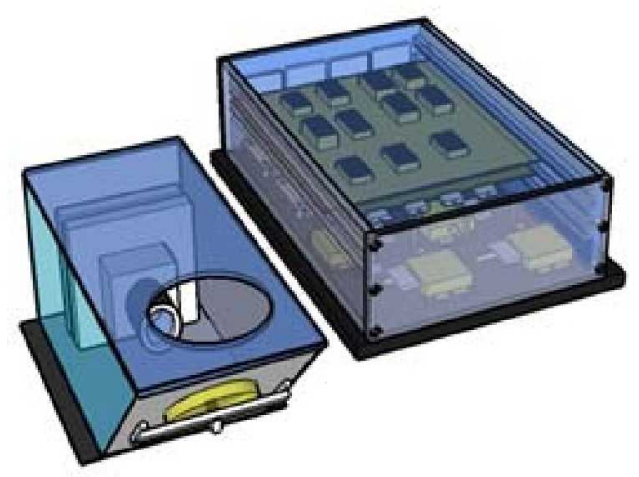

FIGURE 4. NGAVGS Two-Box Concept

During Acquisition or Tracking, image acquisition is initiated by the Digital Signal Processor (DSP). The DSP generates a command to the Field Programmable Gate Array (FPGA) to begin a cycle. The FPGA starts by issuing a fire laser command to the Laser Housekeeping Processor. The lasers are fired and the imager accumulates charge for a predetermined amount of time (the integration time or exposure time). Once the integration time is complete, the image is passed from the imager to the FPGA where it is stored in external memory. Two complete images are accumulated in this manner with each image being stored in separate memory. The differences in the two images are primarily caused by the different reflections of the two different wavelengths of lasers that were fired during the integration time. The two images are then compared by the FPGA, and the lit comparison data is compressed and passed to the DSP where pattern matching is performed. A final solution is calculated and transmitted out thru the RS-422 serial interface. The image can be seen in real time thru the Video Output port. A general block-diagram of the NGAVGS is shown below in Figure 5.

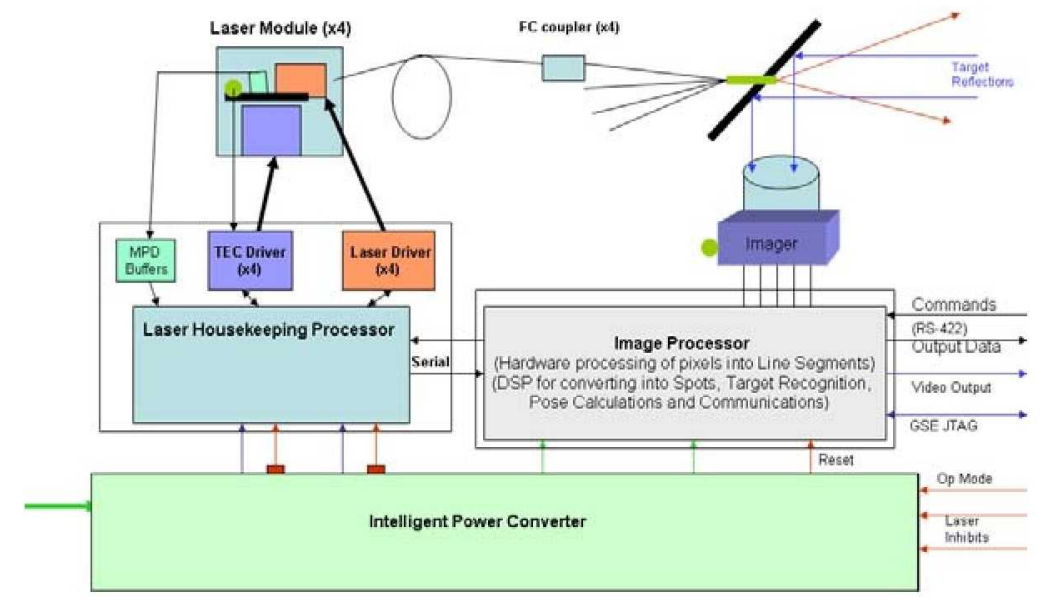

FIGURE 5. Block Diagram showing connections and functions of the NGAVGS

A breadboard version of the NGAVGS was built at MSFC to test the new components that were chosen to replace the obsolete components. Once the breadboard had been successfully tested, two brassboard versions of the NGAVGS were built.

Part of the challenge of upgrading a well performing sensor is confirming any performance changes to the new sensor due to parts that have different performance or configuration. With a new imager and lens and a change in the laser illumination system, the resolution and exposure parameters of the new sensor need to be verified with either old or new target configurations. For the NGAVGS, the Orbital Express Short Range Target and Long Range Target filtered reflectors (OE SRT \& LRT) were selected for the 1 to $200 \mathrm{~m}$ docking range and two International Space Station (ISS) -like hemi-spherical un-filtered reflectors for berthing approaches from $5000 \mathrm{~m}$ into $4 \mathrm{~m}$. 


\section{NGAVGS DEVELOPMENT UNIT TESTING}

The first brassboard was used for testing from ranges of 1 meter to 300 meters. The initial testing consisted primarily of imaging different retro-reflective targets at various ranges and angles to determine the probable overall performance of the initial NGAVGS.

The close-range testing with the OE SRT occurred in the Flight Robotics Laboratory, which has a precise computer controlled a two axis sensor gimbal for sensor azimuth and elevation motion and a three axis target gimbal providing target pitch, yaw, and roll positioning. The repeatability and convenience of this testing capability is used for initial focus, alignment, resolution, and exposure parameter testing for various target angles in the center, edge, and corner of the sensor field of view from $1 \mathrm{~m}$ out to $100 \mathrm{~m}$.

The middle range testing occurred in the 300 meter tunnel facility which has undergone renovations and upgrades of its own to turn it into a closed-loop computerized test capability. The Apollo-Saturn-era $300 \mathrm{~m}$ underground cable tunnel was cleared of $11,000 \mathrm{ft}$ of cable trays and 200,000 to $300,000 \mathrm{ft}$ of multi-conductor instrumentation cable. A four axis Remote Automated Target Transport (RATT) was designed and built. The RATT positions target mockups with desired yaw, pitch, and roll angles and at positions from 5 to $300 \mathrm{~m}$ in front of the sensor. The sensor can be mounted on a two axis gimbal for testing any portion of the sensor's field-of-view. This facility is controlled by a computer that can move all six axes (range, azimuth, elevation, yaw, pitch, and roll) from static position or rate commands, multi-axis multi-step automated scripts, and simple sensor driven closed-loop dynamic testing. The long range testing was performed with various retro-reflectors - an OE type SRT, 1.5 inch diameter OE corner-cube retro-reflectors, and a pair of ISS- like hemispherical arrays of seven 1-inch diameter retro-reflectors spaced apart similar to the pair on the bottom of the Japanese pressurized ISS laboratory module, as seen on the right in Figure 3.

Figure 6 shows the background image captured by the new sensor showing the ceiling lights, then it shows the foreground image with the lights and two reflected spots of the target, and finally it shows the subtracted image with the two very bright spots of the reflectors and very dim outlines of the lights.

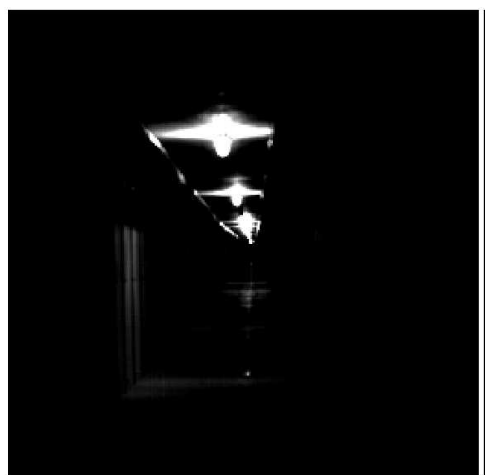

(a)

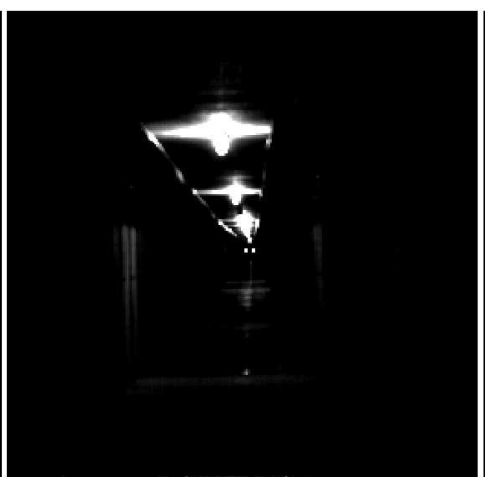

(b)

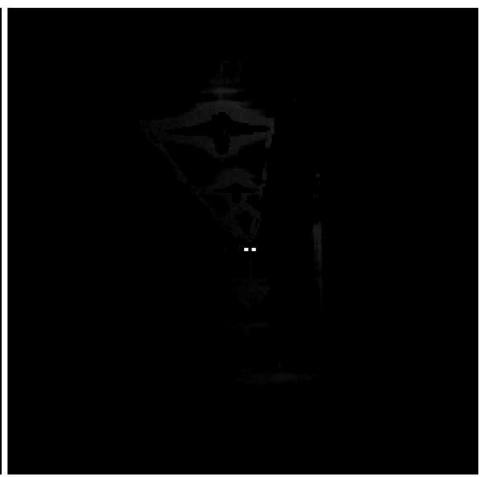

(c)

FIGURE 6. (a) Background Image, (b) Foreground image, and (c) Subtracted image of retro-reflectors at 300 meters.

The second brassboard was used for testing at very long ranges (from 300 meters to 3000 meters). Because there were no available indoor test ranges with the distances required, this testing had to be performed outdoors. Prior to performing the outdoor testing, permission had to be acquired from the Army Redstone Arsenal and from the Federal Aviation Administration (FAA). The very long range testing used Apollo-Saturn-era test stands and towers and the surrounding roadways. This very long range testing was performed with the new sensor and an AVGS EDU shooting out of the eleventh floor window at the top of a test tower and an ISS- like hemispherical array of seven 1inch diameter retro-reflectors mounted on the elevated handrail of a Saturn 5 engine test stand about $2000 \mathrm{~m}$ south of the test tower and another hemi reflector array mounted on the elevated handrail of a Saturn 1 booster test stand about $3000 \mathrm{~m}$ south of the test tower shown in Figure 7.

There was some definite atmospheric interference that caused the spots to vary in intensity and size for both the new sensor and the proven AVGS EDU (from nothing visible to a good, bright spot of about 15 pixels.) The humidity was greater than $80 \%$, and the high humidity and the dense air caused the air to interfere with the laser output and 
the return signal. At $2000 \mathrm{~m}$, the new sensor saw the reflector array with just two lasers at about the same integration time as the AVGS Engineering Demonstration Unit (EDU). However, to see the $3000 \mathrm{~m}$ reflector, the new sensor had to have the maximum integration time, which was several times longer than for $2000 \mathrm{~m}$, to clearly see the spot in the center or the edge of the field of view, possibly due to atmospheric conditions or unknown sensor behavior.

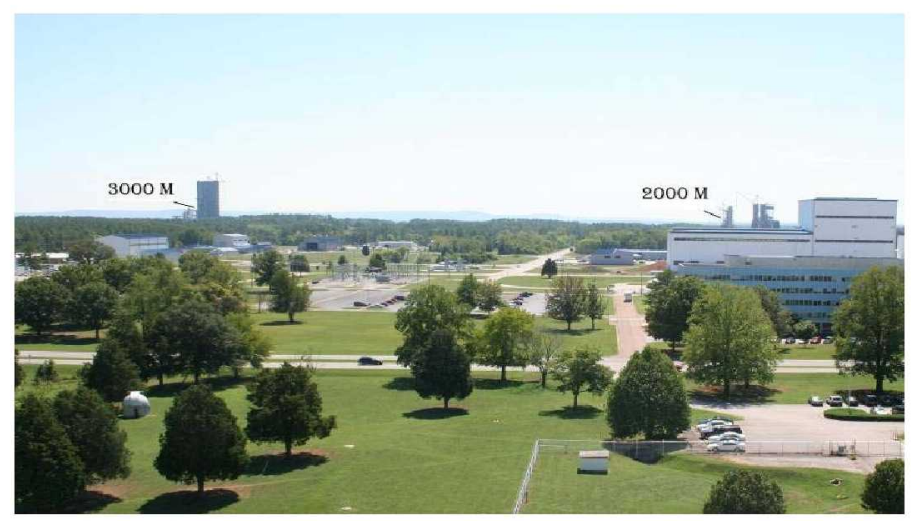

FIGURE 7. South View out of Tower Window

Based on the initial brassboard tests, lens and laser changes are being evaluated in the brassboard and an engineering unit was designed to meet specifications that were based on the Advanced Video Guidance Sensor performance specifications. A brassboard was first built with the updates to the lasers and lens as well as a higher resolution imager, and it was tested at ranges of 3000, 4000, and 5000 meters. Images taken from the sensor during testing at with targets at $4 \mathrm{~km}$ is shown in Figure 8. The results of the testing were positive. The sensor could detect two separate retro-reflectors at each range, it could give a range and bearing reading, and there was laser power and exposure time to spare. That means that there is margin in the optical power of the sensor, and it means that the sensor could probably operate at even longer ranges were that a requirement.

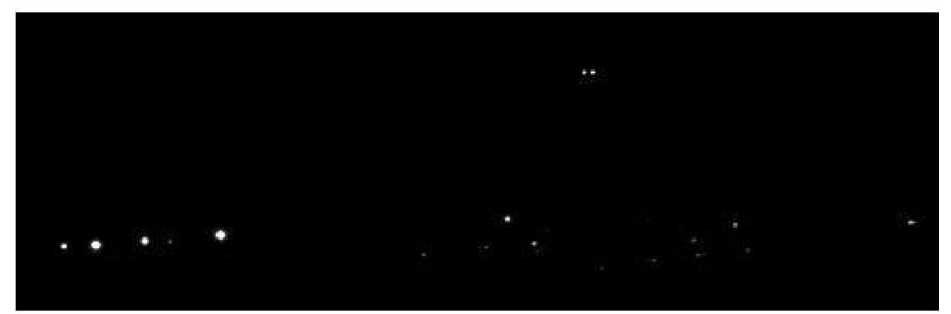

(a)

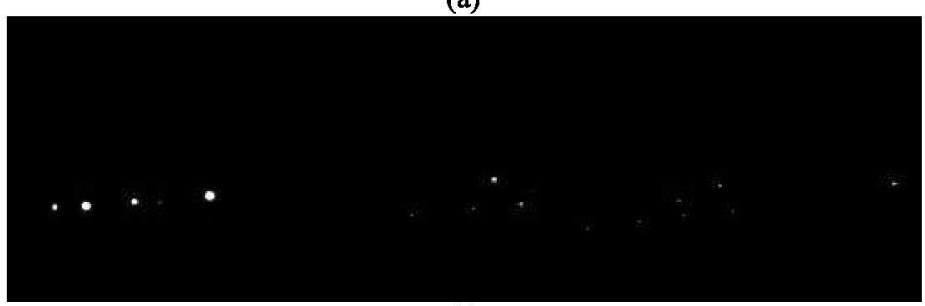

(b)

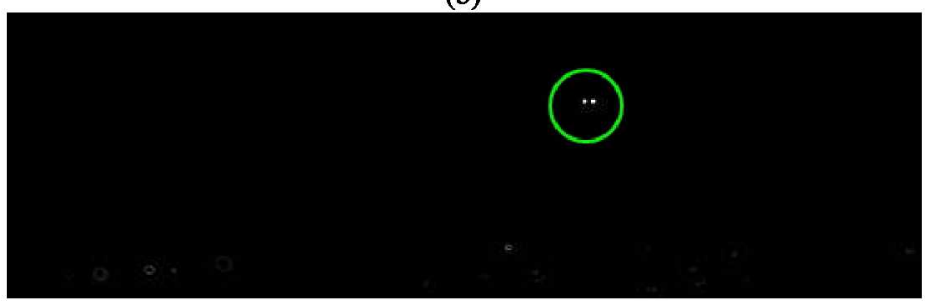

(c)

FIGURE 8. Foreground image (a) (street lights visible along with retro-reflective spots), background image (b) with same lights but no reflected spots, and then the subtracted image (c) with the target spots circled. 
During the course of that testing, an engineering unit was built. The engineering unit was the Block I prototype, and it was tested in the laboratory as well as in the long range test facility at $3 \mathrm{~km}$. It also functioned well, demonstrating that it could see retro-reflectors at that long range, see two distinct retro-reflectors, and actually provide range and bearing data to a pair of retro-reflectors spaced as far apart as some of the retro-reflectors on the ISS.

The baseline NGAVGS performance requirements are similar to the OE AVGS requirements with regard to range of operation and accuracy, but it must be noted that the sensor performance depends heavily on the target configuration used. The NGAVGS, like its predecessors, looks at the spots of light generated by illuminating a retro-reflective target. The accuracy at which the sensor tracks those spots is a function of reflector size, range, and position in the Field of View.

\section{CONCLUSION}

The work performed so far in the design, development, and test of the NGAVGS has been quite successful. The hardware trades resulted in a promising design and the tests have shown that the design was a good one. The NGAVGS benefits from the experiences gained from the successful OE AVGS and it is following in the footsteps of its successful progenitors, the AVGS and the VGS. The NGAVGS is on track to support the Constellation and COTS programs.

\section{ACRONYMS}

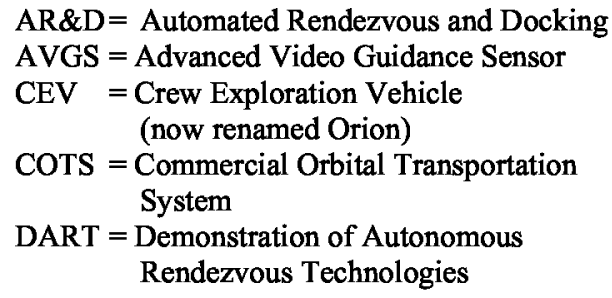

$$
\begin{array}{ll}
\text { EDU } & =\text { Engineering Development Unit } \\
\text { ISS } & =\text { International Space Station } \\
\text { LRT } & =\text { Long Range Target } \\
\text { MSFC } & =\text { Marshall Space Flight Center } \\
\text { NGAVGS } & =\text { Next Generation AVGS } \\
\text { OE } & =\text { Orbital Express } \\
\text { SRT } & =\text { Short Range Target } \\
\text { VGS } & =\text { Video Guidance Sensor }
\end{array}
$$

\section{ACKNOWLEDGMENTS}

The authors give much thanks to the entire team of people working on this sensor. Their dedication and professionalism have allowed this program to be very successful in creating a capable next generation sensor for automated rendezvous and docking.

\section{REFERENCES}

Howard, R. T., Johnston, A. S., Bryan, T. C. and Book, M. L., "Advanced Video Guidance Sensor (AVGS) development testing," in Spaceborne Sensors, edited by R. D. Habbit, Jr. and P. Tchoryk, Jr., SPIE Conference Proceedings, Vol. 5418, Bellingham, WA, (2004), pp. 50-60. 